\title{
Characterizing Cell-Decomposable Metrics
}

\author{
Katharina T. Huber \\ School of Computing Sciences \\ University of East Anglia \\ Norwich, NR4 7TJ, UK \\ katharina.huber@cmp.uea.ac.uk \\ Vincent Moulton \\ School of Computing Sciences \\ University of East Anglia \\ Norwich, NR4 7TJ, UK \\ vincent.moulton@cmp.uea.ac.uk
}

\author{
Jacobus Koolen \\ Department of Mathematics \\ POSTECH \\ Pohang, South Korea \\ koolen@postech.ac.kr \\ Andreas Spillner \\ School of Computing Sciences
University of East Anglia
Norwich, NR4 7TJ, UK
aspillner@cmp.uea.ac.uk
}

Submitted: Aug 14, 2007; Accepted: Mar 6, 2008; Published: Mar 20, 2008

Mathematics Subject Classifications: 54E35, $05 \mathrm{C} 12$

\begin{abstract}
To a finite metric space $(X, d)$ one can associate the so called tight-span $T(d)$ of $d$, that is, a canonical metric space $\left(T(d), d_{\infty}\right)$ into which $(X, d)$ isometrically embeds and which may be thought of as the abstract convex hull of $(X, d)$. Amongst other applications, the tight-span of a finite metric space has been used to decompose and classify finite metrics, to solve instances of the server and multicommodity flow problems, and to perform evolutionary analyses of molecular data. To better understand the structure of $\left(T(d), d_{\infty}\right)$ the concept of a cell-decomposable metric was recently introduced, a metric whose associated tight-span can be decomposed into simpler tight-spans. Here we show that cell-decomposable metrics and totally split-decomposable metrics - a class of metrics commonly applied within phylogenetic analysis - are one and the same thing, and also provide some additional characterizations of such metrics.
\end{abstract}

\section{Introduction}

In this note $(X, d)$ denotes a finite metric space, that is, a finite set $X$ together with a metric or function $d: X \times X \rightarrow \mathbb{R}_{\geq 0}$ such that $d(x, x)=0, d(x, y)=d(y, x)$ and $d(x, z) \leq d(x, y)+d(y, z)$ hold for all $x, y, z \in X$. The tight-span $T(d)$ of $d[6]$ is the polytopal complex consisting of the bounded faces or cells of the polyhedron

$$
P(d):=\left\{f \in \mathbb{R}^{X}: f(x)+f(y) \geq d(x, y) \text { for all } x, y \in X\right\}
$$


(see Section 2 for the definition of a polyhedral complex and related concepts). The pair $\left(T(d), d_{\infty}\right)$, where $d_{\infty}: T(d) \times T(d) \rightarrow \mathbb{R}_{\geq 0}$ is defined, for each $f, g \in T(d)$, by

$$
d_{\infty}(f, g):=\max _{x \in X}|f(x)-g(x)|,
$$

is a metric space into which $(X, d)$ embeds isometrically via the map, that for each $x \in X$, takes $x$ to the function

$$
v_{x}=v_{x}^{X}: X \rightarrow \mathbb{R}_{\geq 0}: y \mapsto d(x, y) .
$$

The tight-span was introduced by J. Isbell [18] in the 1960's, and was subsequently rediscovered in $[3,6]$. More recently, it has also been shown to arise in the context of tropical geometry $[4,5]$. Amongst other applications, the tight-span has been used to decompose and classify metrics [8, 22], solve instances of the server [21] and multicommodity flow $[2,19]$ problems, understand optimal realizations of metrics $[6,10]$, and also as a tool within phylogenetic analysis [13]. Most of these applications heavily rely on the application of structural properties of the tight-span.

To better understand the combinatorial and geometrical structure of the tight-span, in [15] the concept of a cell-decomposable metric was introduced which is defined as follows. Given a cell $C$ of $T(d)$ and some $x \in X$, we call a (necessarily unique) element $\gamma^{x} \in C$ a gate in $C$ for $x$ if, for all $h \in C$,

$$
d_{\infty}\left(v_{x}, h\right)=d_{\infty}\left(v_{x}, \gamma^{x}\right)+d_{\infty}\left(\gamma^{x}, h\right)
$$

We say that $C$ is $X$-gated if there is a gate in $C$ for each $x \in X$. In case every cell in $T(d)$ is $X$-gated, $d$ is called cell-decomposable. Cell-decomposable metrics are so called because their tight-spans can be decomposed into simpler tight-spans [15].

In [15] it was conjectured that a metric $d$ is cell-decomposable if and only if it is totally split-decomposable, that is, defining for any four elements $x, y, u, v \in X$, the quantity

$$
\alpha(x y \mid u v):=\max \{\max \{d(x, u)+d(y, v), d(x, v)+d(y, u)\}-d(x, y)-d(u, v), 0\},
$$

$d$ satisfies

$$
\alpha(x y \mid u v) \leq \alpha(x t \mid u v)+\alpha(x y \mid u t)
$$

for all $t, x, y, u, v \in X$. Totally split-decomposable metrics were introduced by Bandelt and Dress in [1], and are extensively applied within the area of phylogenetic analysis [17].

In [16] it is shown that if a metric is totally split-decomposable then it is cell-decomposable. As a consequence it is also shown that the cells in the tight-span associated to a totally split-decomposable metric are zonotopes that are polytope isomorphic to either hypercubes or rhombic dodecahedra. In this note we prove the following (note that some alternative characterizations of totally split-decomposable metrics are presented in $[1,11,12])$ :

Theorem 1.1 If $d$ is a metric on $X$, then the following assertions are equivalent:

(i) $d$ is totally split-decomposable. 
(ii) $d$ is cell-decomposable.

(iii) every 1-cell of $T(d)$ is $X$-gated.

(iv) every 2-cell of $T(d)$ is $X$-gated.

Intriguingly, if $d$ is a metric for which every $i$-cell of $T(d)$ is $X$-gated, $i \geq 3$, it does not follow that $d$ is totally split-decomposable (see e.g. Figure 1). It would be interesting to characterize those metrics for which every 3 -cell is $X$-gated. More generally, it would be of interest to understand metrics $d$ for which every maximal cell in $T(d)$ (under cell inclusion) is $X$-gated.

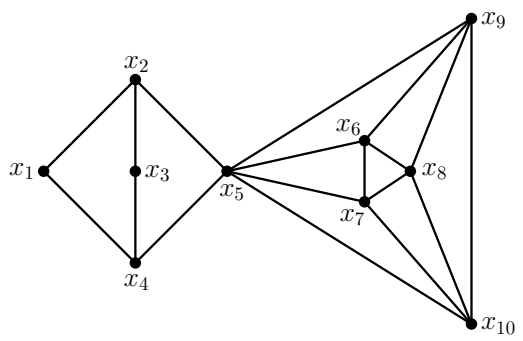

Figure 1: The metric $d$ induced on the vertex set $\left\{x_{1}, x_{2}, \ldots, x_{10}\right\}$ of the graph in this figure (as usual, by taking shortest paths between vertices) is not totally split-decomposable even though the unique 3 -cell in $T(d)$ is $X$-gated.

The rest of this note is organized as follows. In Section 2 we present some preliminaries concerning tight-spans, and in Section 3 we show that $X$-gated cells must satisfy two key combinatorial properties (Propositions 3.1 and 3.3). These are then used in Section 4 to prove Theorem 1.1.

\section{Preliminaries}

In this section, we present some useful results on the tight-span of a finite metric space.

We first recall some definitions concerning polyhedral complexes from [20]. For $n \geq 1$ an integer, a polyhedron in $\mathbb{R}^{n}$ is the intersection of a finite collection of halfspaces in $\mathbb{R}^{n}$ and a polytope is a bounded polyhedron. A face of a polyhedron $P$ is the empty-set, $P$ itself, or the intersection of $P$ with a supporting hyperplane and, if $P$ is $i$-dimensional, $i \geq 1$, then its 0 -, 1 - and $(i-1)$-dimensional faces are called its vertices, edges and facets, respectively. The collection of all faces of a polytope forms a lattice with respect to the ordering given by set inclusion. We say that two polytopes are polytope isomorphic if their face-lattices are isomorphic. A polytopal complex $\mathcal{C}$ is a finite collection of polytopes such that each face of a member of $\mathcal{C}$ is itself a member of $\mathcal{C}$, and the intersection of two members of $\mathcal{C}$ is a face of each. We call the members of $\mathcal{C}$ cells and an $i$-dimensional cell an $i$-cell, for short.

Now, for $f$ a function in $P(d)$, we define the tight-equality graph of $f, K(f)=$ $(X, E(f))$, to be the graph with vertex set $X$ and edge set $E(f)$ consisting of those 
$\{x, y\} \subseteq X$ with $f(x)+f(y)=d(x, y)$. Note that $E(f)$ might contain loops. By $[f]$ we denote the minimal face $C$ in $P(d)$ that contains $f$. If $C$ is a cell in $T(d)$ and $f \in C$ with $C=[f]$, we call $f$ a generator of $C$. Since the graph $K(f)$ is the same for every generator $f$ of $C$ we denote this graph also by $K(C)$.

Using these definitions, we now present some useful properties of $T(d)$, proofs for which can be found in $[6,7,14]$ :

(T0) $d_{\infty}\left(v_{x}, f\right)=f(x)$ for every $x \in X$ and every $f \in T(d)$.

(T1) $[f]=\{g \in T(d): E(f) \subseteq E(g)\}$ for every $f \in T(d)$.

(T2) $K(C)$ is a proper subgraph of $K\left(C^{\prime}\right)$ if and only if $C^{\prime} \subsetneq C$, for all cells $C, C^{\prime}$ in $T(d)$.

(T3) The number of connected components of $K(C)$ that are bipartite equals $\operatorname{dim}(C)$, the dimension of the cell $C$, for every cell $C$ in $T(d)$.

We conclude this section with two observations concerning $T(d)$. The first observation concerns a property of tight-equality graphs.

Lemma 2.1 Suppose $d$ is a metric on $X, f \in T(d)$ such that $\operatorname{dim}[f] \geq 1$, and $Y$ is the vertex set of a non-bipartite connected component of $K(f)$. Then for all $x \in Y$ and all $h \in[f]$, we have $h(x)=f(x)$.

Proof: Suppose $f \in T(d)$ with $\operatorname{dim}[f] \geq 1$ and $Y$ is the vertex set of a non-bipartite connected component of $K(f)$. Then, by the definition of the tight-equality graph, every edge $e=\{x, y\} \subseteq Y$ of $K(f)$ corresponds to an equation $E_{e}$, namely $f(x)+f(y)=d(x, y)$. Suppose $h \in[f]$. By $(\mathrm{T} 1), K(f)$ is a subgraph of $K(h)$. Thus, we also have $h(x)+h(y)=$ $d(x, y)$. Hence, the set of equations $\left\{E_{e} \mid e \subseteq Y\right.$ an edge of $\left.K(f)\right\}$ holds for any $h \in[f]$. But since the connected component on $Y$ is not bipartite it must contain an odd cycle and, so, the solution to this set of equations must be unique. Thus, $h(x)=f(x)$ holds for every $h \in[f]$ and every $x \in Y$.

In our second observation we present a simple but useful relationship between the structure of the tight-equality graph $K(C)$ for a cell $C$ of $T(d)$ and the values of $f$ for $f \in C$.

Lemma 2.2 Suppose that $d$ is a metric on $X$ and $C$ is a cell of $T(d)$. Let $\pi$ denote the path $x=w_{1}, w_{2}, \ldots, w_{l}=y$ in $K(C)$. Then there exists some real number $a_{y}$ such that for every $f \in C$ holds

$$
f(y)= \begin{cases}a_{y}+f(x) & \text { if path } \pi \text { has even length } \\ a_{y}-f(x) & \text { if path } \pi \text { has odd length }\end{cases}
$$


Proof: Consider any $f \in C$. By property (T1) for every $k \in\{1, \ldots, l-1\}$ the edge $\left\{w_{k}, w_{k+1}\right\}$ is contained in $E(f)$ and, thus, $d\left(w_{k}, w_{k+1}\right)=f\left(w_{k}\right)+f\left(w_{k+1}\right)$. Hence, depending on the parity of the length of $\pi$ we can express either $f(y)-f(x)$ or $f(y)+f(x)$ in terms of $d\left(w_{1}, w_{2}\right), \ldots, d\left(w_{l-1}, w_{l}\right)$ only, which is independent of $f$.

\section{The tight-equality graph of an $X$-gated cell}

In this section, we prove that if $d$ is a metric on $X$ and $C$ is an $X$-gated $i$-cell of $T(d)$, $i \geq 1$, then $K(C)$ is bipartite (Proposition 3.1), and, moreover, if $i>1$ also holds, then $K\left(C^{\prime}\right)$ is bipartite for every $(i-1)$-cell $C^{\prime} \subsetneq C$ (Proposition 3.3).

Proposition 3.1 Let $d$ be a metric on $X$ and suppose that $C$ is an $X$-gated $i$-cell of $T(d), i \geq 1$. Then $K(C)$ is bipartite.

Proof: Suppose $C$ is an $i$-cell of $T(d), i \geq 1$. We show that if $K(C)$ is not bipartite then $C$ is not $X$-gated. So, suppose $K(C)$ is not bipartite. Then there is at least one connected component of $K(C)$ that is not bipartite. Let $Y$ denote the vertex set of one such component. Then, by Lemma 2.1, $h(x)=f(x)$ holds for all $x \in Y$ and all $f, h \in C$. Consequently, by (T0),

$$
d_{\infty}\left(v_{x}, h\right)=h(x)=f(x)=d_{\infty}\left(v_{x}, f\right) .
$$

Now assume for contradiction that $C$ is $X$-gated and fix some arbitrary element $x \in Y$. Then there must exist some $\gamma^{x} \in C$ such that

$$
d_{\infty}\left(v_{x}, h\right)=d_{\infty}\left(v_{x}, \gamma^{x}\right)+d_{\infty}\left(\gamma^{x}, h\right)
$$

holds for every $h \in C$. Since $\operatorname{dim}(C) \geq 1$, we may choose some $\hat{h} \in C$ distinct from $\gamma^{x}$. Fix some generator $f$ of $C$. By Equations (1) and (2),

$$
\begin{aligned}
d_{\infty}\left(v_{x}, f\right)=d_{\infty}\left(v_{x}, \hat{h}\right) & =d_{\infty}\left(v_{x}, \gamma^{x}\right)+d_{\infty}\left(\gamma^{x}, \hat{h}\right) \\
& =d_{\infty}\left(v_{x}, f\right)+d_{\infty}\left(\gamma^{x}, \hat{h}\right)>d_{\infty}\left(v_{x}, f\right)
\end{aligned}
$$

follows which is impossible.

Note that there are metrics for which the converse of Proposition 3.1 does not hold. To prove Proposition 3.3, we shall use the following lemma concerning gates of an $X$-gated cell.

Lemma 3.2 Let $x \in X$ and let $C$ denote an $X$-gated cell of $T(d)$. Then $h \in C$ is the gate of $x$ in $C$ if and only if

$$
d_{\infty}\left(v_{x}, h\right)=\min \left\{d_{\infty}\left(v_{x}, h^{\prime}\right) \mid h^{\prime} \in C\right\} .
$$


Proof: Let $x \in X$ and assume that $C$ is $X$-gated. Suppose first that $h \in C$ such that $d_{\infty}\left(v_{x}, h\right)=\min \left\{d_{\infty}\left(v_{x}, h^{\prime}\right) \mid h^{\prime} \in C\right\}$. Since $C$ is $X$-gated, there exists some map $\gamma^{x} \in C$ that is the gate for $x$ in $C$. Hence,

$$
d_{\infty}\left(v_{x}, \gamma^{x}\right) \geq d_{\infty}\left(v_{x}, h\right)=d_{\infty}\left(v_{x}, \gamma^{x}\right)+d_{\infty}\left(\gamma^{x}, h\right) \geq d_{\infty}\left(v_{x}, \gamma^{x}\right)
$$

and thus $h=\gamma^{x}$ must hold.

Conversely suppose $\gamma^{x} \in C$ is a gate for $x$ in $C$ and assume to the contrary that $d_{\infty}\left(v_{x}, \gamma^{x}\right)>\min \left\{d_{\infty}\left(v_{x}, h^{\prime}\right) \mid h^{\prime} \in C\right\}$. Now choose $h \in C$ with the property that $d_{\infty}\left(v_{x}, h\right)=\min \left\{d_{\infty}\left(v_{x}, h^{\prime}\right) \mid h^{\prime} \in C\right\}$. Then $d_{\infty}\left(v_{x}, h\right)<d_{\infty}\left(v_{x}, \gamma^{x}\right)$ and so

$$
d_{\infty}\left(v_{x}, h\right)+d_{\infty}\left(\gamma^{x}, h\right)<d_{\infty}\left(v_{x}, \gamma^{x}\right)+d_{\infty}\left(\gamma^{x}, h\right)=d_{\infty}\left(v_{x}, h\right)
$$

since $\gamma^{x}$ is the gate for $x$ in $C$. But then $d_{\infty}\left(\gamma^{x}, h\right)<0$ which is impossible.

Proposition 3.3 Let $d$ be a metric on $X$ and let $C$ denote an $X$-gated $i$-cell of $T(d)$, $i \geq 2$. Then for every $(i-1)$-cell $C^{\prime} \subseteq C$ the graph $K\left(C^{\prime}\right)$ is bipartite.

Proof: Suppose $C$ is some $X$-gated $i$-cell of $T(d), i \geq 2$. By Proposition 3.1, $K(C)$ is bipartite and, thus, we can color the vertices of $K(C)$ with two different colors so that no two adjacent vertices are colored the same.

Consider the vertex set $Y$ of any connected component of $K(C)$. By Lemma 2.2, for every $x, y \in Y$ there exists some $a_{y} \in \mathbb{R}$ such that for all $h \in C$ we have

$$
h(y)= \begin{cases}a_{y}+h(x) & \text { if } x \text { and } y \text { have the same color } \\ a_{y}-h(x) & \text { if } x \text { and } y \text { have different colors. }\end{cases}
$$

We claim that there is no $g \in C, \operatorname{dim}[g] \geq 1$, such that there is an edge $e$ of $K(g)$ which is not an edge of $K(C)$ and both endpoints of $e=\{x, y\}$ belong to the same connected component of $K(C)$. Suppose for contradiction that such an element $g \in C$ exists. Fix an arbitrary generator $f$ of $C$.

Note first that $x$ and $y$ must share the same color. Indeed, if $x$ and $y$ were colored differently then, on the one hand, $d(x, y)=g(x)+g(y)=a_{y}=f(x)+f(y)$, by equation (3). But $e$ is not an edge of $K(f)$ and thus, on the other hand, $f(x)+f(y)>d(x, y)$ holds. But this is impossible and so $x$ and $y$ must be colored with the same color. In consequence, the connected component of $K(g)$ that contains vertices $x$ and $y$ must contain an odd cycle, and is, thus, not bipartite. So, by Lemma 2.1,

$$
d_{\infty}\left(v_{x}, g^{\prime}\right)=g^{\prime}(x)=g(x)
$$

follows for all $g^{\prime} \in[g]$.

Now, to complete the proof of the claim, consider any $h \in C$. By the definition of $T(d)$ and (3), we obtain

$$
h(x)+h(y) \geq d(x, y) \text { and } h(y)=a_{y}+h(x) .
$$


Similarly

$$
g(x)+g(y)=d(x, y) \text { and } g(y)=a_{y}+g(x)
$$

follows. Combining these four equations yields $2 h(x) \geq d(x, y)-a_{y}=2 g(x)$ and, thus, $h(x) \geq g(x)$ must hold. Consequently,

$$
\min \{h(x) \mid h \in C\}=g(x)
$$

and thus, by (T0) and (4),

$$
\min \left\{d_{\infty}\left(v_{x}, h\right) \mid h \in C\right\}=\min \{h(x) \mid h \in C\}=g^{\prime}(x)
$$

for all $g^{\prime} \in[g]$. By Lemma 3.2, every map $g^{\prime} \in[g]$ must be a gate for $x$ in $C$. But then, by the uniqueness of a gate, $[g]$ must be a vertex which is impossible. This establishes the claim.

To conclude the proof assume that $C^{\prime} \subseteq C$ is an $(i-1)$-cell of $T(d)$. Then, since $\operatorname{dim}(C)=i$, by (T2) the graph $K(C)$ must be a proper subgraph of $K\left(C^{\prime}\right)$. Choose some edge $e$ of $K\left(C^{\prime}\right)$ that is not contained in $K(C)$. Then, by the previous claim, the endpoints of $e$ must belong to different connected components of $K(C)$. Furthermore, since the dimension of $C^{\prime}$ is one less than the dimension of $C$, (T3) implies that the number of bipartite connected components of $K\left(C^{\prime}\right)$ must be one less than the number of bipartite connected components of $K(C)$. Since, as was observed at the beginning of the proof, $K(C)$ is bipartite and $e$ already connects two different connected components of $K(C)$, all connected components of $K\left(C^{\prime}\right)$ must be bipartite. Hence, $K\left(C^{\prime}\right)$ is bipartite.

\section{Proof of Theorem 1.1}

In the proof we shall use the fact that $d$ is totally split-decomposable if and only if for every 1-cell $C$ of $T(d)$ the graph $K(C)$ is bipartite [11, Proposition 3.2]. We refer to this latter property as condition (v), in addition to conditions (i) - (iv) listed in the statement of Theorem 1.1.

Now, in [16, p. 475] it was shown that (i) implies (ii). Moreover, by definition, if $d$ is cell-decomposable then every 1-cell and every 2-cell of $T(d)$ must be $X$-gated. Thus, trivially, (ii) implies (iii) and (iv).

We now show that (iii) implies (v). Assume that every 1-cell of $T(d)$ is $X$-gated. Then, by Proposition 3.1, for every 1-cell $C$ of $T(d)$ the graph $K(C)$ is bipartite, which established (v).

To finish the proof we show that (iv) implies (v). Suppose that every 2-cell of $T(d)$ is $X$-gated. Consider any 1-cell $C^{\prime}$ of $T(d)$. If $C^{\prime}$ is maximal then $K\left(C^{\prime}\right)$ is bipartite. To see this note first that $T(d) \backslash\{f\}$ is disconnected for any $f$ in the relative interior of $C^{\prime}$. Thus, by [9, Theorem 1], the graph $K\left(C^{\prime}\right)$ is connected. Hence, by (T3), $K\left(C^{\prime}\right)$ is bipartite. If $C^{\prime}$ is contained in a 2-cell $C$ then, by Proposition 3.3, $K\left(C^{\prime}\right)$ is bipartite. Hence, for every 1-cell $C^{\prime}$ of $T(d)$ the graph $K\left(C^{\prime}\right)$ is bipartite. 
Acknowledgment: The authors would like to thank the Engineering and Physical Sciences Research Council (EPSRC) for its support (grant EP/D068800/1).

\section{References}

[1] H. J. Bandelt and A. Dress. A canonical decomposition theory for metrics on a finite set. Advances in Mathematics, 92:47-105, 1992.

[2] V. Chepoi. A $T_{X}$-approach to some results on cuts and metrics. Advances in Applied Mathematics, 19:453-470, 1997.

[3] M. Chrobak and L. Larmore. Generosity helps or an 11-competitive algorithm for three servers. Journal of Algorithms, 16:234-263, 1994.

[4] M. Develin and B. Sturmfels. Tropical convexity. Documenta Mathematica, 9:1-27, 2004.

[5] M. Develin and B. Sturmfels. Erratum for "tropical convexity". Documenta Mathematica, 9:205-206, 2004.

[6] A. Dress. Trees, tight extensions of metric spaces, and the cohomological dimension of certain groups: A note on combinatorial properties of metric spaces. Advances in Mathematics, 53:321-402, 1984.

[7] A. Dress. Towards a classification of transitive group actions on finite metric spaces. Advances in Mathematics, 74:163-189, 1989.

[8] A. Dress, K. T. Huber, J. Koolen, and V. Moulton. Six points suffice: how to check for metric consistency. European Journal of Combinatorics, 22:465-474, 2001.

[9] A. Dress, K. T. Huber, J. Koolen, and V. Moulton. Cut points in metric spaces. Applied Mathematics Letters, 2007. in press.

[10] A. Dress, K. T. Huber, J. Koolen, V. Moulton, and A. Spillner. A note on metric cut points and bridges, 2007. submitted.

[11] A. Dress, K. T. Huber, A. Lesser, and V. Moulton. Hereditarily optimal realizations of consistent metrics. Annals of Combinatorics, 10:63-76, 2006.

[12] A. Dress, K. T. Huber, and V. Moulton. A comparison between two distinct continuous models in projective cluster theory: The median and the tight-span construction. Annals of Combinatorics, 2:299-311, 1998.

[13] A. Dress, K. T. Huber, and V. Moulton. An explicit computation of the injective hull of certain finite metric spaces in terms of their associated buneman complex. Advances in Mathematics, 168:1-28, 2002.

[14] A. Dress, V. Moulton, and W. Terhalle. T-theory: An overview. European Journal of Combinatorics, 17:161-175, 1996.

[15] K. T. Huber, J. Koolen, and V. Moulton. The tight-span of an antipodal metric space: Part II - geometrical properties. Discrete and Computational Geometry, 31:567-586, 2004. 
[16] K. T. Huber, J. H. Koolen, and V. Moulton. On the structure of the tight-span of a totally split-decomposable metric. European Journal of Combinatorics, 27:461-479, 2006.

[17] D. H. Huson and D. Bryant. Application of phylogenetic networks in evolutionary studies. Molecular Biology and Evolution, 23:254-267, 2006.

[18] J. Isbell. Six theorems about metric spaces. Commentarii Mathematici Helvetici, 39:65-74, 1964.

[19] A. Karzanov. Metrics with finite sets of primitive extensions. Annals of Combinatorics, 2:211-241, 1998.

[20] V. Klee and P. Kleinschmidt. Convex polytopes and related complexes. In R. L. Graham, M. Grötschel, and L. Lovász, editors, Handbook of Combinatorics, Part I. Elsevier, 1999.

[21] L. Larmore and J. Oravec. T-theory applications to online algorithms for the server problem, 2006. arXiv:cs/0611088v1.

[22] B. Sturmfels and J. Yu. Classification of six-point metrics. The Electronic Journal of Combinatorics, 11, 2004. 